\title{
Prioritization of Sub-Watersheds in Ruparel Watershed, Rajasthan Based on Morphometric and Land Use/Land Cover Analysis Using Remote Sensing and GIS
}

\author{
Munahzah Meraj*, Akram Javed \\ Department of Geology, Aligarh Muslim University, Aligarh, India \\ Email: ^munzzim9@gmail.com, akramjaved70@gmail.com
}

How to cite this paper: Meraj, M. and Javed, A. (2022) Prioritization of Sub-Watersheds in Ruparel Watershed, Rajasthan Based on Morphometric and Land Use/Land Cover Analysis Using Remote Sensing and GIS. International Journal of Geosciences, 13, 138-154.

https://doi.org/10.4236/ijg.2022.132008

Received: January 4, 2022

Accepted: February 25, 2022

Published: February 28, 2022

Copyright $\odot 2022$ by author(s) and Scientific Research Publishing Inc. This work is licensed under the Creative Commons Attribution International License (CC BY 4.0).

http://creativecommons.org/licenses/by/4.0/ (c) (i) Open Access

\begin{abstract}
Watershed prioritization is considered as the most significant aspect in watershed resource management and development program. The present work attempts to prioritize seventeen sub-watersheds in Ruparel watershed of Alwar district of Rajasthan, India. For prioritization of sub-watersheds, morphometric and land use/land cover (LULC) analysis were performed using remote sensing and GIS. Base map of the study area has been derived from SOI toposheet on 1:50,000 scale whereas LULC mapping was done using IRS P6 LISS III data. Standard methods for drainage morphometry have been followed for computing morphometric parameters such as linear and shape for seventeen sub-watersheds and allotted ranks based on their relationship with erodibility and a compound value has been calculated for final ranking. Five main LULC categories were computed and were assigned priority ranks and subsequently a compound parameter was determined for final ranking. Integration of both morphometric and LULC results reveal that SBW5, SBW7, SBW12 and SBW16 are the common sub-watersheds that fall under high priority, SBW3 falls under Medium category and SBW11 comes under low priority. The results of the analysis can be used to identify the sub-watersheds which need immediate restoration and will eventually help in watershed resource management for sustainable development.
\end{abstract}

\section{Keywords}

Prioritization, Watershed Resource Management, Morphometry, Land Use/Land Cover Analysis 


\section{Introduction}

Natural resources like land, forest and water are finite, their improper and uncontrolled consumption is posing a serious threat to the precious environment particularly in developing countries like India, hence their conservation and proper management is essential for sustainable development. Watershed is considered as an ideal unit for proper management and effective planning of land, forest and water resources by classifying it into smaller units, i.e., sub-watersheds or milli-watersheds by taking into consideration various terrain conditions such as contour values, drainage network, relief and spot height [1].

Watershed prioritization classifies different watersheds in order of their priority so that management and conservation measures can be implemented. Morphometric analysis with the help of remote sensing and GIS techniques is considered to be the most useful approach for prioritization of watersheds [2]-[10]. Morphometry allows the quantitative analysis of a drainage basin which is an important aspect in the characterization of watersheds [11]. Sub-watersheds can be prioritized on the basis of a number of factors such as drainage basin morphometry, Universal Soil Loss Equation (USLE), Sediment Yield Index (SYI), LULC analysis etc. [1] [3] [12]. LULC mapping is considered as an important component in which other characteristics are combined on the requirement basis to drive various developmental indexes for land and water resources [13]. Drainage basin morphometry together with soil and LULC analysis is very critical for developing a water resource action plan that includes locating recharge and discharge zones as well as for classification and prioritization of the watersheds [6]. A host of workers have attempted watershed prioritization in different parts of India with the help of remote sensing and GIS [1] [3] [4] [5] [8] [13]. The current study prioritizes seventeen sub-watersheds in Ruparel watershed of Alwar district of Rajasthan using techniques such as morphometric and LULC analysis with the help of remote sensing and GIS. The main objectives of this study are to identify and classify sub-watersheds in the Ruparel watershed for conservation of natural resources for ultimate sustainable development and to recommend watershed conservation measures for planning and resource development.

\section{Study Area Description}

Ruparel watershed is located in Alwar district of Rajasthan, India and occupies an area of $1215.76 \mathrm{~km}^{2}$. The watershed lies between $27^{\circ} 10^{\prime} \mathrm{N}$ to $27^{\circ} 54^{\prime} \mathrm{N}$ latitudes and $76^{\circ} 16^{\prime} \mathrm{E}$ and $76^{\circ} 45^{\prime} \mathrm{E}$ longitudes and the maximum and minimum elevation of the watershed is $732 \mathrm{~m}$ and $231 \mathrm{~m}$ above mean sea level (MSL), respectively. The study area falls in Alwar, Bansur, Thanghazi, and Rajgarh tehsils of Alwar district. Agriculture is the primary economic activity in the study area and the major crops grown are bajra, wheat, gram, Gaur, groundnut, arhar and mustard. The main Ruparel River also known as Barah or Laswari Nadi originates from Udainath hills in Thanaghazi tehsil of Alwar district, transverses northwards 
passing through the Sariska forest and then flows eastwards from Natni ka Bara where from an $8 \mathrm{kms}$ long feeder channel known as Barah Feeding Channel diverts the Alwar share of water to Jaisamand lake. The Ruparel River continues to flow eastwards, passes through the fertile plains of the Alwar district and finally terminates in the adjoining Bharatpur district (Figure 1). The Ruparel watershed is mainly rainfed and experiences semi-arid climate. Watershed shows dendritic to sub-dendritic drainage pattern in general but locally trellis, rectangular and parallel patterns have also developed.

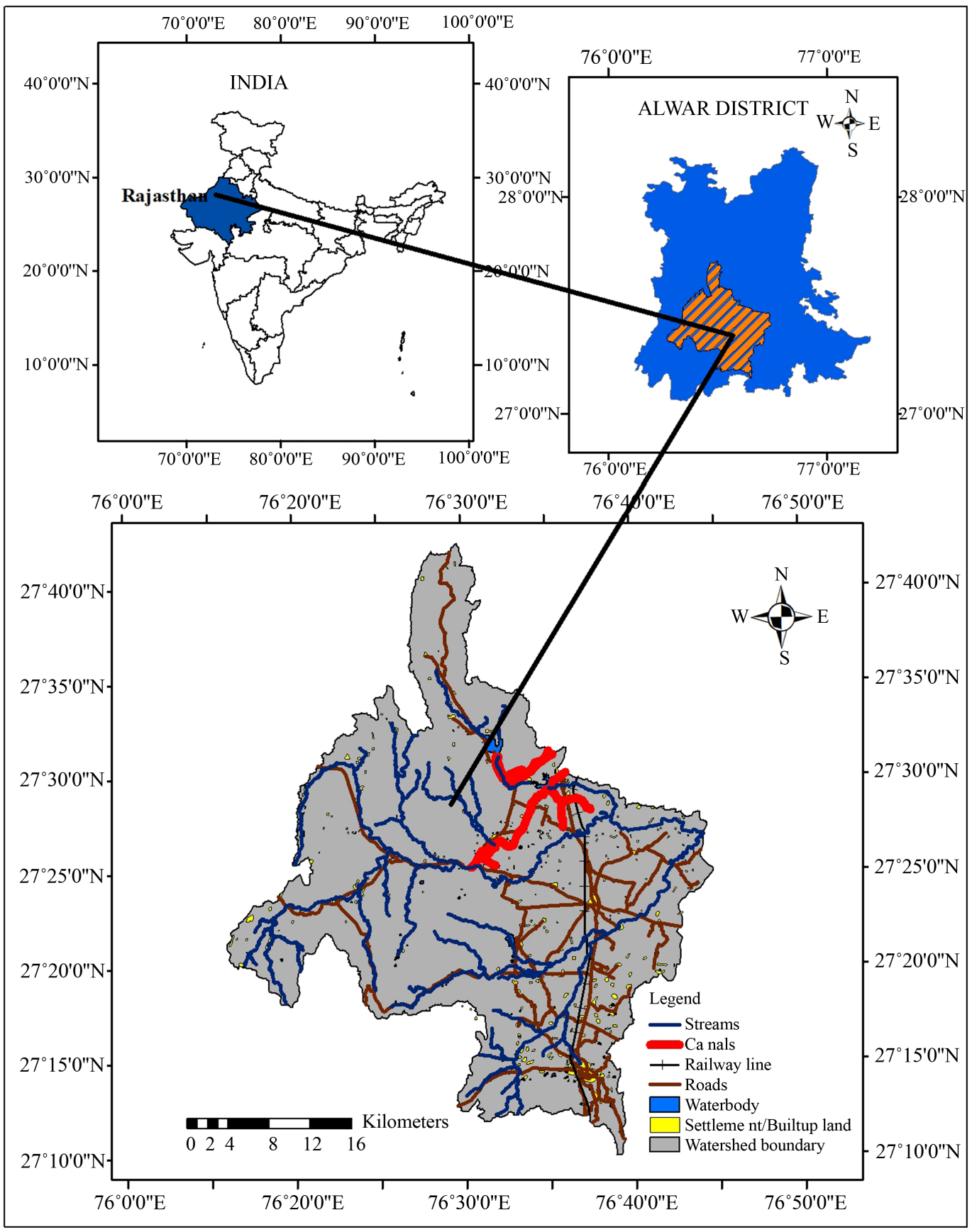

Figure 1. Location map of Ruparel watershed. 


\section{Data Used and Methodology}

The present study has utilised Survey of India (SOI) toposheets 54A/6, 54A/7, $54 \mathrm{~A} / 8,54 \mathrm{~A} / 10,54 \mathrm{~A} / 11$ and $54 \mathrm{~A} / 12$ on $1: 50,000$ scale. The toposheets were scanned, mosaiced and then georeferenced and registered to UTM projection (WGS 84, zone 43) in ARC GIS environment for using as baseline data of the study area. The watershed boundary was delineated by considering first order channels, contour lines, spot height and drainage divide. SOI toposheets have been utilized to generate the drainage network of the study area by on screen digitization using ArcGIS 10.5 software (Figure 2). Elevation map of the study

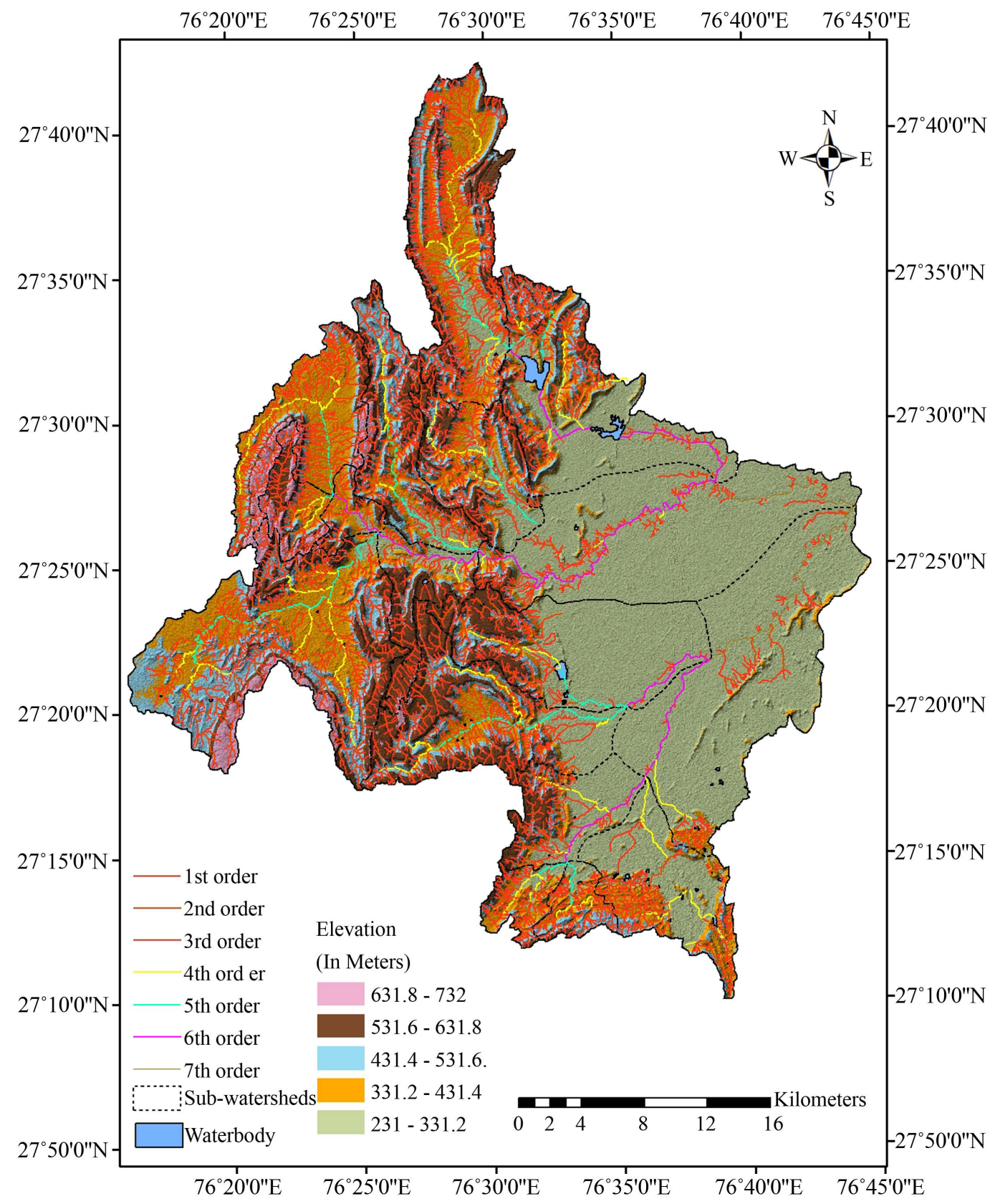

Figure 2. Drainage network superimposed on the elevation map of the study area. 
area was produced from Advanced Space borne Thermal Emission Radiometer data of $30 \mathrm{~m}$ resolution downloaded from USGS website

(https://earthexplorer.usgs.gov/) (Figure 2). The Ruparel watershed was classified into 17 sub-watersheds and named as SBW1, SBW2, SBW3, SBW4 and so on. Smallest SBW i.e., SBW3 occupies an area of $12.67 \mathrm{~km}^{2}$ whereas largest SBW i.e., SBW1 occupies an area of $154.76 \mathrm{~km}^{2}$. For each sub-watershed quantitative morphometric parameters such as bifurcation ratio, drainage density, stream frequency, drainage texture, length of overland flow, basin shape, form factor, elongation ratio, circularity ratio and length of overland flow were computed using standard formulae and methods [11] [14] [15] [16] [17] [18].

Satellite data IRS LISS III FCC (False colour composite) of 2014 (Path-Row: 95 - 52) was utilized to identify various LULC categories. Seventeen LULC categories were identified and delineated using visual image interpretation methods and include fallow land, cultivated land, dense forest, open forest, degraded forest, open scrub, dense scrub, ravenous land (with open scrub), ravenous land, exposed rock (with open scrub), settlement/build up land, waterbody, dry waterbody, plantation, barren land, barren/rocky/stony waste, and stone quarry. LULC analysis in terms of area and percentage under each LULC category was done sub-watershed wise using ArcGIS. Morphometric and LULC analysis were used as basic elements for carrying out prioritization in Ruparel watershed. Morphometric parameters such as linear and shape parameters were calculated and priority ranking was given based on their relationship with erodibility [4] [5]. For prioritization based on LULC analysis, categories such as cultivated land, dense forest, open forest, open scrub and wasteland were considered. Based on average priority ranking value, the sub-watersheds were given four priority ranks as Very High, High, Medium and Low.

\section{Results and Discussion}

\subsection{Morphometric Analysis}

The quantitative morphometric characterization of Ruparel watershed was done through the measurement of linear and shape parameters. The first step in the morphometric analysis of a drainage basin is the designation of stream order $(\mathrm{u})$, using hierarchic ranking method of streams [11]. The whole Watershed has been classified into 17 sub-watersheds (viz. SBW1, SBW2, SBW3 etc.) (Figure 2). The mean bifurcation value in the Ruparel watershed ranges from 2.82 to 7 (Table 1). The lower mean bifurcation value in SBW11 shows lack of structural control on drainage development whereas higher value in SBW3 suggests that the drainage pattern is structurally controlled. The values of drainage density range between 0.62 (SBW11) and 4.28 (SBW13) $\mathrm{km} / \mathrm{km}^{2}$ (Table 1). High drainage density values are recorded in SBW13 and SBW14 which represent impermeable sub-surface material, high mountain relief, increased runoff and decreased infiltration capacity whereas moderate drainage density values are observed in SBW4, SBW5, SBW6, SBW7 and SBW8. Low drainage density values are 
Table 1. Morphometric analysis of the Ruparel watershed.
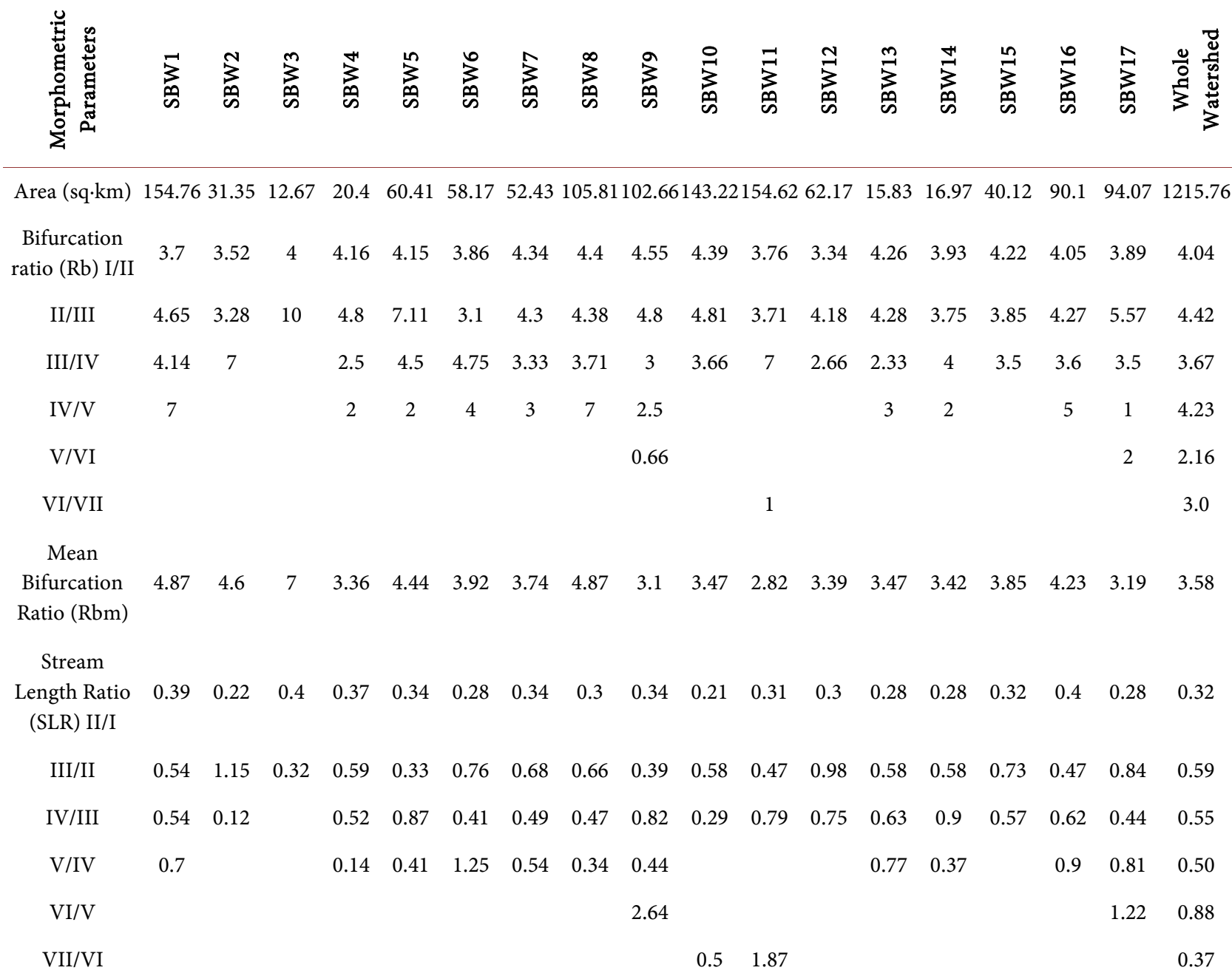

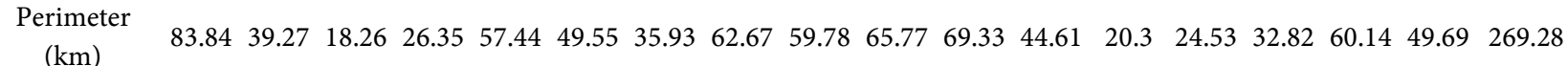

Basin

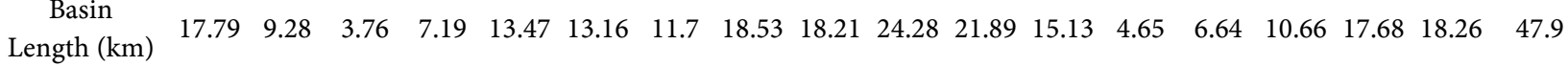

Drainage

$\begin{array}{lllllllllllllllllll}\text { Density } & 2.84 & 2.56 & 2.85 & 3.55 & 3.72 & 3.3 & 3.08 & 3.9 & 2.11 & 1.09 & 0.62 & 2.03 & 4.28 & 4.1 & 2.33 & 2.9 & 1.37 & 2.32\end{array}$ $\mathrm{D}\left(\mathrm{km} / \mathrm{km}^{2}\right)$

Stream

$\begin{array}{lllllllllllllllllll}\text { Frequency } & 4.34 & 3.6 & 4.1 & 6.47 & 5.66 & 5.34 & 4.67 & 6.14 & 4.13 & 2.1 & 0.86 & 5.03 & 10.68 & 9.36 & 3.76 & 4.58 & 2.15 & 3.93\end{array}$ (Fs)

Drainage Texture (Rt) $\begin{array}{llllllllllllllllll}8.01 & 2.87 & 2.85 & 5 & 5.95 & 6.27 & 6.81 & 10.37 & 7.1 & 4.59 & 1.93 & 7.01 & 8.32 & 6.48 & 4.6 & 6.86 & 4.08 & 17.75\end{array}$

Form Factor (Rf) $\begin{array}{llllllllllllllllll}0.48 & 0.36 & 0.89 & 0.38 & 0.33 & 0.33 & 0.38 & 0.3 & 0.3 & 0.24 & 0.32 & 0.27 & 0.73 & 0.38 & 0.35 & 0.28 & 0.28 & 0.52\end{array}$

Circularity Ratio (Rc) $\begin{array}{llllllllllllllllll}0.27 & 0.25 & 0.47 & 0.39 & 0.23 & 0.29 & 0.51 & 0.33 & 0.36 & 0.41 & 0.4 & 0.39 & 0.48 & 0.35 & 0.46 & 0.31 & 0.47 & 0.21\end{array}$

Elongation Ratio (Re) $\begin{array}{llllllllllllllllll}0.78 & 0.68 & 1.06 & 0.7 & 0.65 & 0.65 & 0.7 & 0.62 & 0.62 & 0.55 & 0.64 & 0.58 & 0.97 & 0.7 & 0.67 & 0.6 & 0.59 & 0.82\end{array}$ 


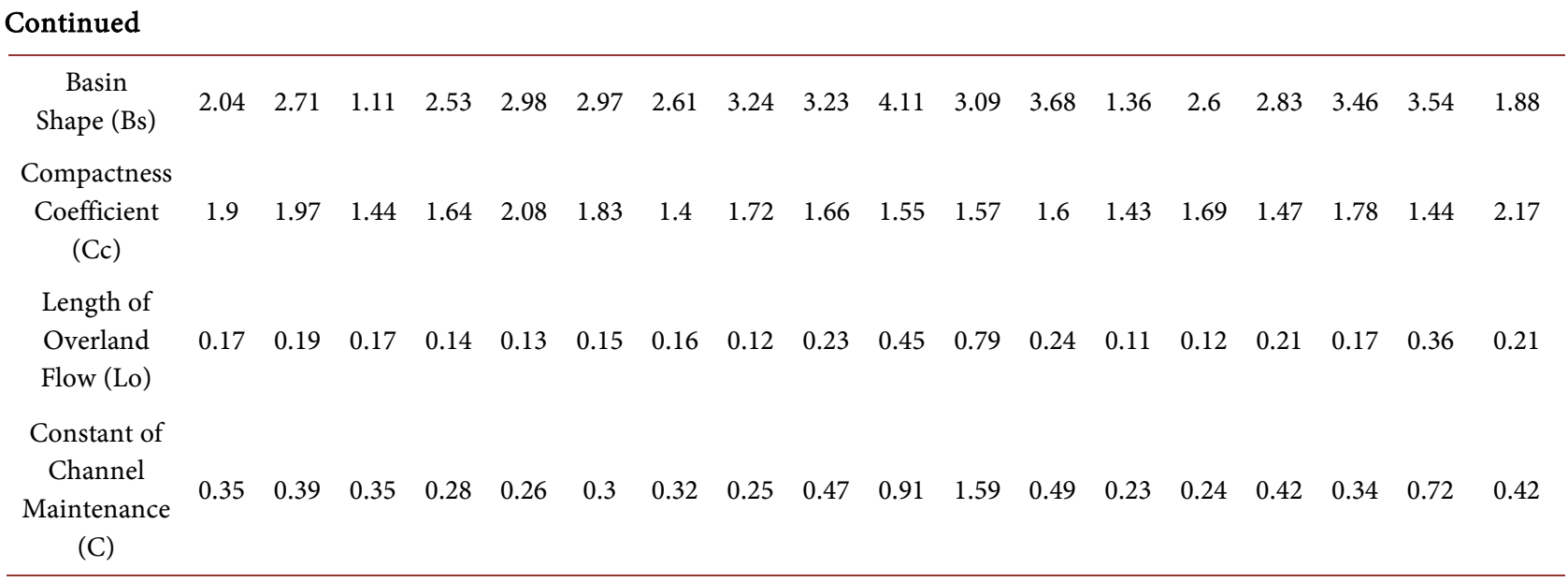

reported from SBW1, SBW2, SBW3, SBW9, SBW10, SBW11, SBW12, SBW15, SBW16 and SBW17 indicating permeable sub soil, low relief, decreased runoff and increased infiltration capacity. Drainage texture values of sub-watersheds range from 1.93 (SBW11) to 10.37 (SBW8). SBW2 and SBW3 represent coarse drainage texture, whereas SBW6, SBW7, SBW9, SBW12, SBW14 and SBW16 display a fine drainage texture. SBW8 is the only sub-watershed which shows very fine drainage texture, whereas remaining of sub-watersheds indicate moderate drainage texture (Table 1). Fine drainage texture is generally observed in sub-watersheds which are characterized by soft and impermeable sub soil material with high basin relief and higher susceptibility to erosion.

The values of Basin shape in sub-watersheds lie between 1.11 (SBW3) and 4.11 (SBW10) (Table 1), which suggest that SBW3 and SBW13 have sharply peaked flood discharge whereas the other 15 sub-watersheds show low flood discharge periods. Circularity ratio values within Ruparel watershed range from 0.23 for (SBW5) to 0.51 (SBW7), indicating overall elongated shape of the sub-watersheds with low runoff and high infiltration due to presence of permeable subsoil material. Lower $(\mathrm{Rc})$ values indicate that the basin is less circular and the discharge rate will be slow and hence the possibility of erosion will be less [6]. Elongation ratio values range from 0.55 (SBW10) to 1.06 (SBW3). Only two sub-watersheds i.e., SBW3 and SBW13 are found to be circular in shape whereas the remaining 15 sub-watersheds fall into elongated to less elongated category. Higher Re values in SBW3 and SBW13 suggest increased infiltration and decreased runoff; whereas, lower Re values in remaining 15 sub-watersheds indicate strong erosive and sediment-load susceptibility.

\subsection{Land Use and Land Cover Analysis}

Seventeen land use/land cover classes (LULC) were identified in Ruparel watershed by visual image interpretation of IRS P6 LISS III FCC imagery of 2014 using various image interpretation elements such as tone, texture, pattern, size, association etc., [19]. LULC categories within the Ruparel watershed include Cultivated land, fallow land, dense forest, open forest, degraded forest, open 
scrub, dense scrub, ravenous land (with open scrub), ravenous land, exposed rock (with open scrub), settlement/build up land, waterbody, dry waterbody, plantation, barren land, barren/rocky/stony waste, and stone quarry (Figure 3 ). LULC statistics has been derived from LULC map by computing area and percentage under each category of LULC (Table 2).

Five main LULC categories such as Cultivated land, Dense Forest, Open Forest, Open scrub and wasteland were taken for the prioritization of sub-watersheds as adopted by [5]. The most dominant land cover category in the Ruparel watershed is Dense Forest, which occupies an area of $373.6 \mathrm{~km}^{2}$ (30.73\%). Cultivated land is the second most dominant category comprising $342.67 \mathrm{~km}^{2}$ (28.19\%) of the watershed area. Wasteland covers $143.48 \mathrm{~km}^{2}(11.8 \%)$ of the total watershed area and comprises ravenous land, ravenous land (with open scrub), barren land, barren/rocky/stony waste and degraded forestland [20]. Open forest covers an area of $33.66 \mathrm{~km}^{2}$ i.e. (2.77\%). Open scrub is covering an area of $70.13 \mathrm{~km}^{2}$ i.e., (5.77\%).

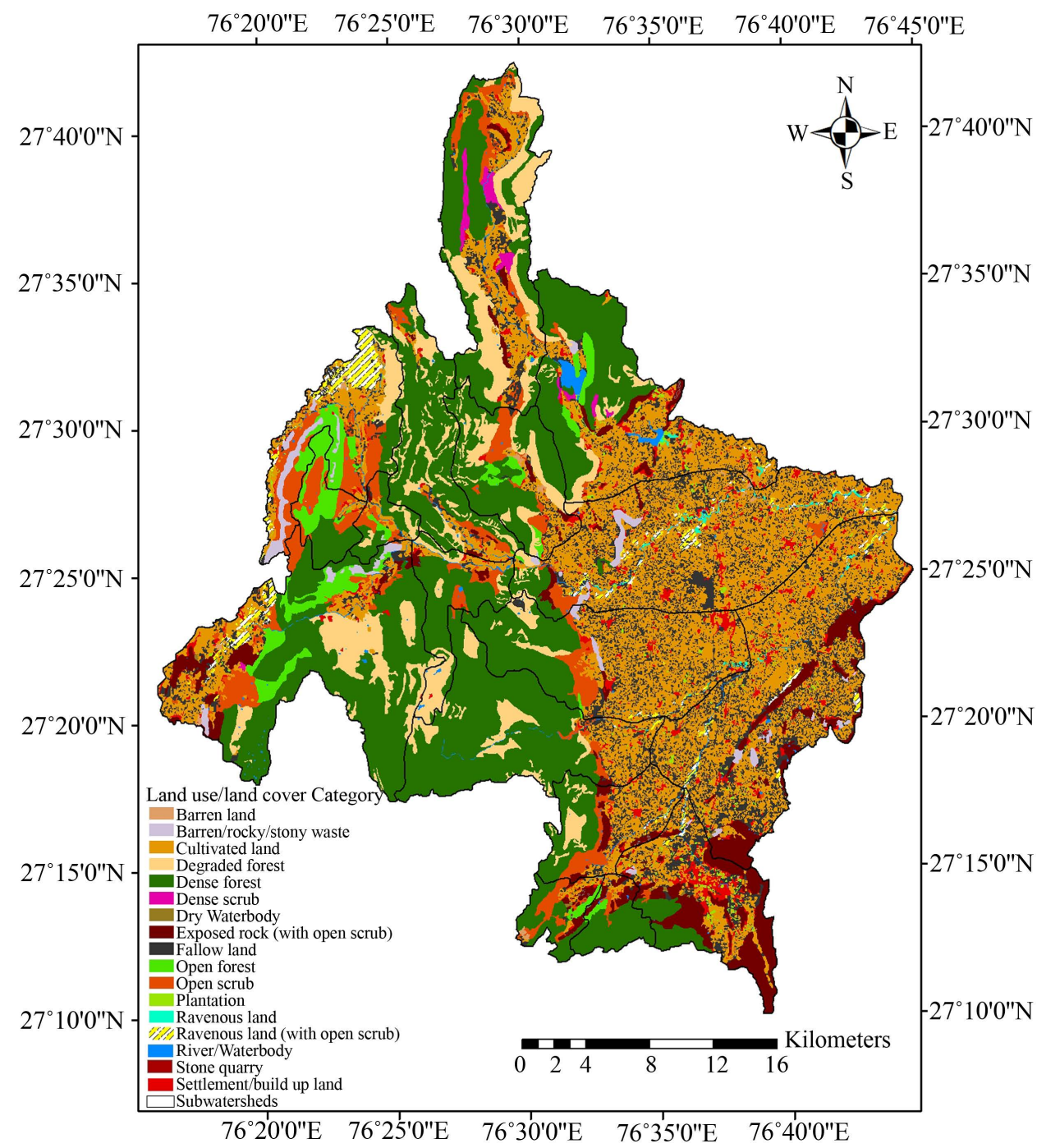

Figure 3. LULC map of Ruparel watershed based on IRS P6 LISS III data of 2014. 
Table 2. Sub-watershed wise LULC analysis of Ruparel watershed.

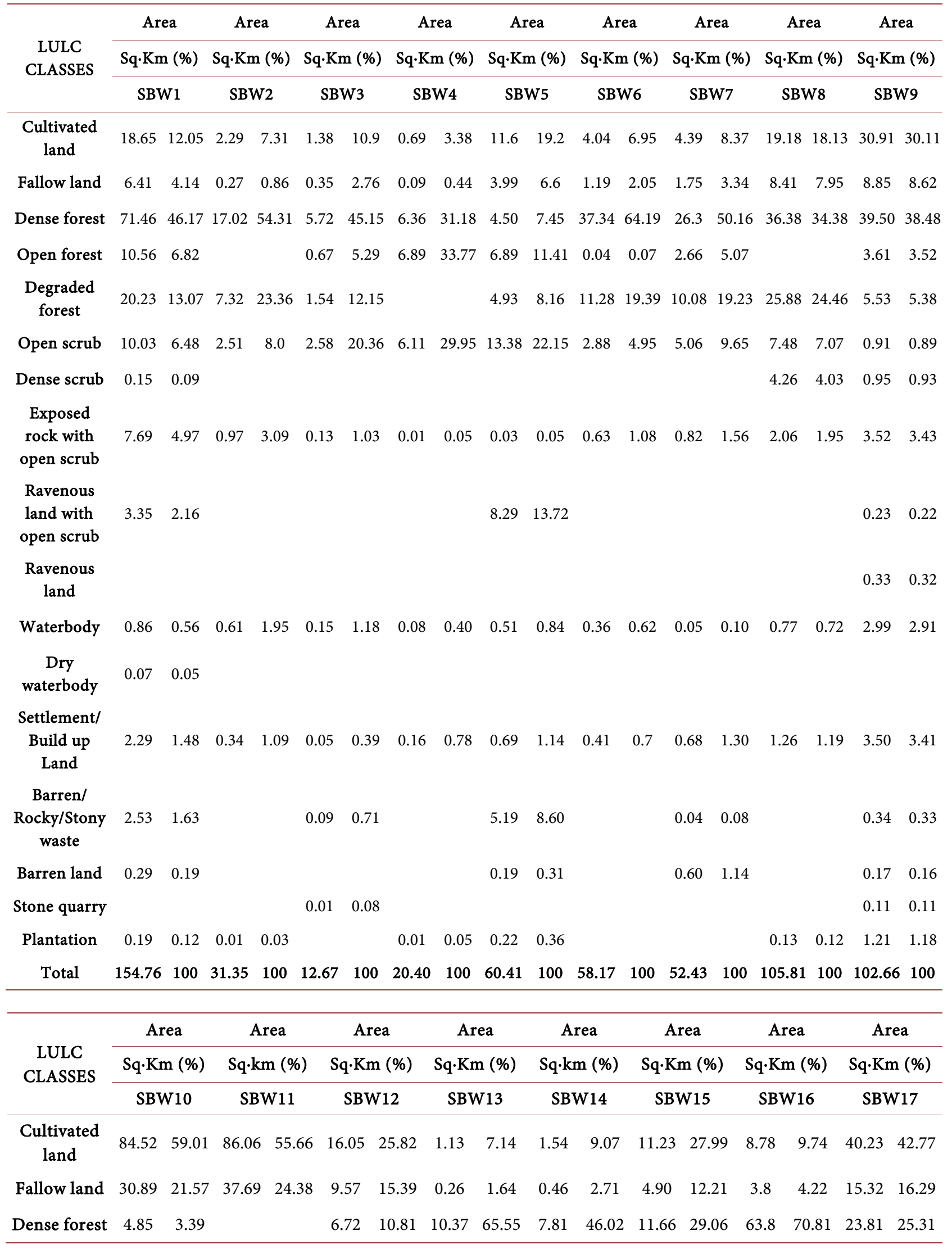




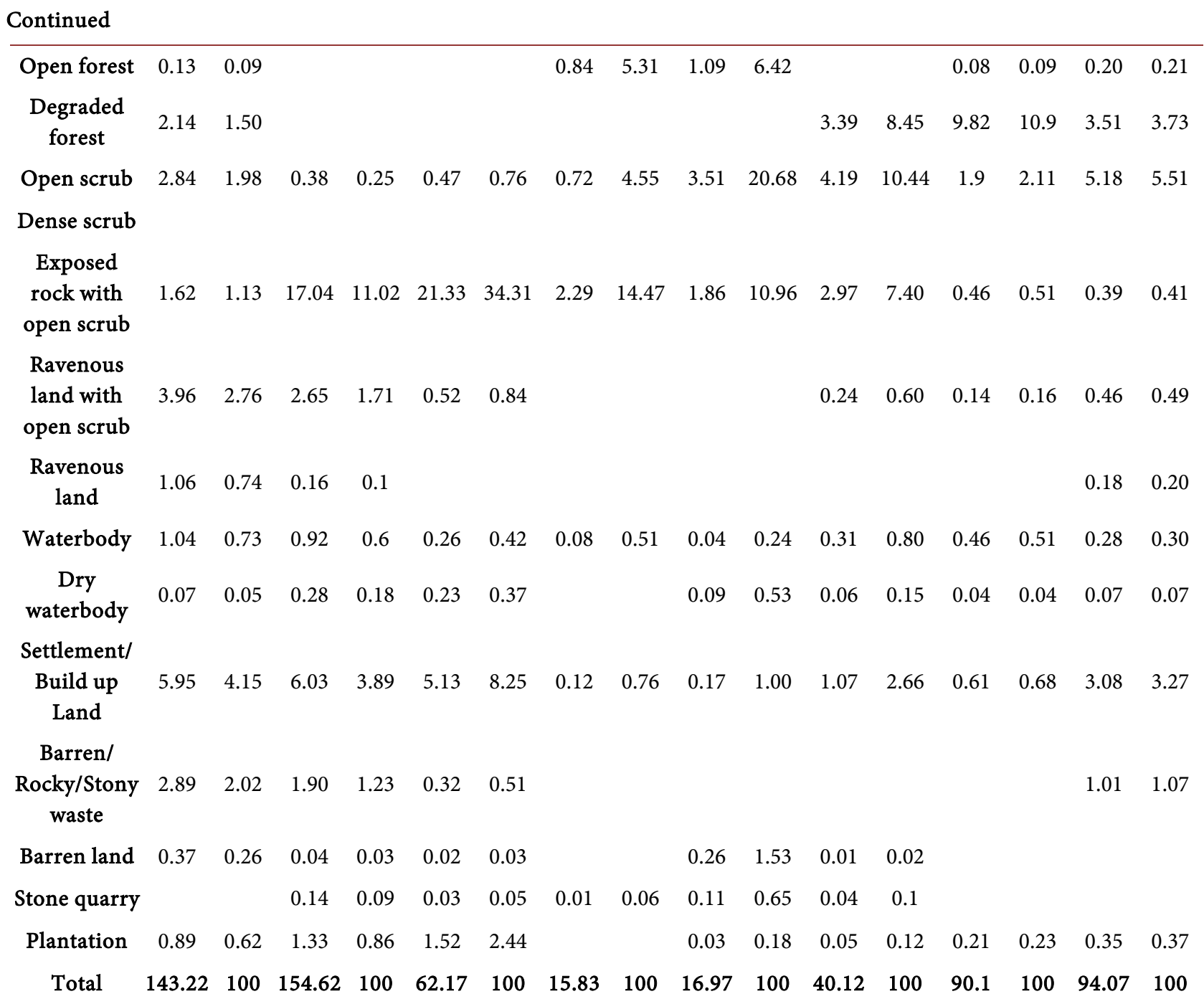

\subsection{Prioritization of Sub-Watersheds Based on Morphometric Parameters}

The morphometric parameters like linear and shape are also termed as Erosion risk assessment parameters [3] and have been taken for the prioritization of sub-watersheds. The linear parameters with direct relationship with erodibility like drainage density, stream frequency, mean bifurcation ratio, and length of overland flow were used for priority ranking. Shape parameters show an inverse relationship with the erodibility i.e. circularity ratio, elongation ratio, form factor and compactness coefficient and were also considered in the ranking process [4].

Hence highest priority ranking was given to the linear parameters with the highest values whereas lowest ranking to the shape parameters with the lowest values.

A compound parameter $(\mathrm{Cp})$ was computed by averaging the rankings for each linear and shape parameters. The sub-watersheds with lowest $(\mathrm{Cp})$ value were given highest piority, next lowest value was assigned second priority and so on and the sub-watersheds were categorized into Very High, High, Medium and 
Low priority classes (Table 3). The results of the analysis show SBW8 and SBW13 fall in the Very High priority category, SBW1, SBW4, SBW5, SBW6, SBW7, SBW12, SBW14 and SBW16 in High priority, SBW2, SBW3, SBW9, SBW10 and SBW15 fall in medium priority whereas SBW11 and SBW17 fall under low priority category (Figure 4 ).

Table 3. Sub-watershed wise priority ranking based on Morphometric analysis.

\section{Morphometric Parameters}

\begin{tabular}{|c|c|c|c|c|c|c|c|c|c|c|c|c|}
\hline \multicolumn{6}{|c|}{ Linear parameters } & \multicolumn{7}{|c|}{ Shape parameters } \\
\hline Sub-watersheds & $\mathrm{D}$ & Fs & $\mathrm{Rbm}$ & Lo & $\mathbf{R t}$ & $\operatorname{Re}$ & $\mathrm{Rc}$ & $\mathrm{Rf}$ & Bs & $\mathrm{Cc}$ & $\mathrm{Cp}$ & Priority value \\
\hline $\begin{array}{c}\text { SBW1 } \\
{[154.76]}\end{array}$ & $\begin{array}{l}2.84 \\
{[10]}\end{array}$ & $\begin{array}{l}4.34 \\
{[10]}\end{array}$ & $\begin{array}{l}4.87 \\
{[2]}\end{array}$ & $\begin{array}{r}0.17 \\
{[8]}\end{array}$ & $\begin{array}{r}8.01 \\
{[3]}\end{array}$ & $\begin{array}{l}0.78 \\
{[11]}\end{array}$ & $\begin{array}{r}0.27 \\
{[3]}\end{array}$ & $\begin{array}{l}0.48 \\
{[10]}\end{array}$ & $\begin{array}{r}2.04 \\
{[3]}\end{array}$ & $\begin{array}{c}1.9 \\
{[14]}\end{array}$ & 7.4 & High \\
\hline $\begin{array}{c}\text { SBW2 } \\
{[31.35]}\end{array}$ & $\begin{array}{l}2.56 \\
{[11]}\end{array}$ & $\begin{array}{l}3.6 \\
{[14]}\end{array}$ & $\begin{array}{l}4.6 \\
{[3]}\end{array}$ & $\begin{array}{r}0.19 \\
\text { [7] }\end{array}$ & $\begin{array}{l}2.87 \\
{[15]}\end{array}$ & $\begin{array}{r}0.68 \\
\text { [9] }\end{array}$ & $\begin{array}{r}0.25 \\
{[2]}\end{array}$ & $\begin{array}{r}0.36 \\
{[8]}\end{array}$ & $\begin{array}{r}2.71 \\
{[7]}\end{array}$ & $\begin{array}{l}1.97 \\
{[15]}\end{array}$ & 9.1 & Medium \\
\hline $\begin{array}{c}\text { SBW3 } \\
{[12.67]}\end{array}$ & $\begin{array}{r}2.85 \\
\text { [9] }\end{array}$ & $\begin{array}{c}4.1 \\
{[12]}\end{array}$ & $\begin{array}{l}7.0 \\
{[1]}\end{array}$ & $\begin{array}{r}0.17 \\
{[8]}\end{array}$ & $\begin{array}{l}2.84 \\
{[16]}\end{array}$ & $\begin{array}{l}1.06 \\
{[13]}\end{array}$ & $\begin{array}{l}0.47 \\
{[13]}\end{array}$ & $\begin{array}{l}0.89 \\
{[12]}\end{array}$ & $\begin{array}{r}1.11 \\
{[1]}\end{array}$ & $\begin{array}{r}1.44 \\
{[3]}\end{array}$ & 8.8 & Medium \\
\hline $\begin{array}{l}\text { SBW4 } \\
{[20.4]}\end{array}$ & $\begin{array}{r}3.55 \\
{[5]}\end{array}$ & $\begin{array}{r}6.47 \\
{[3]}\end{array}$ & $\begin{array}{l}3.36 \\
{[12]}\end{array}$ & $\begin{array}{l}0.14 \\
{[11]}\end{array}$ & $\begin{array}{l}5.0 \\
{[11]}\end{array}$ & $\begin{array}{l}0.7 \\
{[10]}\end{array}$ & $\begin{array}{r}0.39 \\
\text { [9] }\end{array}$ & $\begin{array}{r}0.38 \\
\text { [9] }\end{array}$ & $\begin{array}{r}2.53 \\
{[4]}\end{array}$ & $\begin{array}{r}1.64 \\
{[8]}\end{array}$ & 8.2 & High \\
\hline $\begin{array}{c}\text { SBW5 } \\
{[60.41]}\end{array}$ & $\begin{array}{r}3.72 \\
{[4]}\end{array}$ & $\begin{array}{r}5.66 \\
{[5]}\end{array}$ & $\begin{array}{r}4.44 \\
{[4]}\end{array}$ & $\begin{array}{l}0.13 \\
{[12]}\end{array}$ & $\begin{array}{l}5.95 \\
{[10]}\end{array}$ & $\begin{array}{r}0.65 \\
{[7]}\end{array}$ & $\begin{array}{r}0.23 \\
{[1]}\end{array}$ & $\begin{array}{r}0.33 \\
{[6]}\end{array}$ & $\begin{array}{l}2.98 \\
{[10]}\end{array}$ & $\begin{array}{l}2.08 \\
{[16]}\end{array}$ & 7.5 & High \\
\hline $\begin{array}{c}\text { SBW6 } \\
{[58.17]}\end{array}$ & $\begin{array}{l}3.3 \\
{[6]}\end{array}$ & $\begin{array}{r}5.34 \\
{[6]}\end{array}$ & $\begin{array}{r}3.92 \\
{[6]}\end{array}$ & $\begin{array}{l}0.15 \\
{[10]}\end{array}$ & $\begin{array}{r}6.27 \\
\text { [9] }\end{array}$ & $\begin{array}{r}0.65 \\
{[7]}\end{array}$ & $\begin{array}{r}0.29 \\
{[4]}\end{array}$ & $\begin{array}{r}0.33 \\
{[6]}\end{array}$ & $\begin{array}{r}2.97 \\
\text { [9] }\end{array}$ & $\begin{array}{l}1.83 \\
{[13]}\end{array}$ & 7.6 & High \\
\hline $\begin{array}{c}\text { SBW7 } \\
{[52.43]}\end{array}$ & $\begin{array}{r}3.08 \\
{[7]}\end{array}$ & $\begin{array}{r}4.67 \\
{[8]}\end{array}$ & $\begin{array}{r}3.74 \\
{[8]}\end{array}$ & $\begin{array}{r}0.16 \\
\text { [9] }\end{array}$ & $\begin{array}{r}6.81 \\
{[7]}\end{array}$ & $\begin{array}{l}0.7 \\
{[10]}\end{array}$ & $\begin{array}{l}0.51 \\
{[15]}\end{array}$ & $\begin{array}{r}0.38 \\
\text { [9] }\end{array}$ & $\begin{array}{r}2.61 \\
{[6]}\end{array}$ & $\begin{array}{l}1.4 \\
{[1]}\end{array}$ & 8.0 & High \\
\hline $\begin{array}{c}\text { SBW8 } \\
{[105.81]}\end{array}$ & $\begin{array}{l}3.9 \\
{[3]}\end{array}$ & $\begin{array}{r}6.14 \\
{[4]}\end{array}$ & $\begin{array}{r}4.87 \\
{[2]}\end{array}$ & $\begin{array}{l}0.12 \\
{[13]}\end{array}$ & $\begin{array}{c}10.37 \\
{[1]}\end{array}$ & $\begin{array}{r}0.62 \\
{[5]}\end{array}$ & $\begin{array}{r}0.33 \\
{[6]}\end{array}$ & $\begin{array}{l}0.3 \\
{[4]}\end{array}$ & $\begin{array}{l}3.24 \\
{[12]}\end{array}$ & $\begin{array}{l}1.72 \\
{[11]}\end{array}$ & 6.1 & Very High \\
\hline $\begin{array}{c}\text { SBW9 } \\
{[102.66]}\end{array}$ & $\begin{array}{l}2.11 \\
{[13]}\end{array}$ & $\begin{array}{l}4.13 \\
{[11]}\end{array}$ & $\begin{array}{l}3.1 \\
{[14]}\end{array}$ & $\begin{array}{r}0.23 \\
{[5]}\end{array}$ & $\begin{array}{l}7.1 \\
{[4]}\end{array}$ & $\begin{array}{r}0.62 \\
{[5]}\end{array}$ & $\begin{array}{r}0.36 \\
{[8]}\end{array}$ & $\begin{array}{l}0.3 \\
{[4]}\end{array}$ & $\begin{array}{l}3.24 \\
{[12]}\end{array}$ & $\begin{array}{r}1.66 \\
\text { [9] }\end{array}$ & 8.5 & Medium \\
\hline $\begin{array}{c}\text { SBW10 } \\
{[143.22]}\end{array}$ & $\begin{array}{l}1.09 \\
{[16]}\end{array}$ & $\begin{array}{l}2.1 \\
{[16]}\end{array}$ & $\begin{array}{r}3.47 \\
\text { [9] }\end{array}$ & $\begin{array}{r}0.45 \\
{[2]}\end{array}$ & $\begin{array}{l}4.59 \\
{[13]}\end{array}$ & $\begin{array}{r}0.55 \\
{[1]}\end{array}$ & $\begin{array}{l}0.41 \\
{[11]}\end{array}$ & $\begin{array}{r}0.24 \\
{[1]}\end{array}$ & $\begin{array}{l}4.11 \\
{[16]}\end{array}$ & $\begin{array}{r}1.55 \\
{[5]}\end{array}$ & 9.0 & Medium \\
\hline $\begin{array}{l}\text { SBW11 } \\
{[154.62]}\end{array}$ & $\begin{array}{l}0.62 \\
{[17]}\end{array}$ & $\begin{array}{l}0.86 \\
{[17]}\end{array}$ & $\begin{array}{l}2.82 \\
{[15]}\end{array}$ & $\begin{array}{r}0.79 \\
{[1]}\end{array}$ & $\begin{array}{l}1.93 \\
{[17]}\end{array}$ & $\begin{array}{r}0.64 \\
{[6]}\end{array}$ & $\begin{array}{c}0.4 \\
{[10]}\end{array}$ & $\begin{array}{r}0.32 \\
{[5]}\end{array}$ & $\begin{array}{l}3.09 \\
{[11]}\end{array}$ & $\begin{array}{r}1.57 \\
{[6]}\end{array}$ & 10.5 & Low \\
\hline $\begin{array}{l}\text { SBW12 } \\
{[62.17]}\end{array}$ & $\begin{array}{l}2.03 \\
{[14]}\end{array}$ & $\begin{array}{r}5.03 \\
{[7]}\end{array}$ & $\begin{array}{l}3.39 \\
{[11]}\end{array}$ & $\begin{array}{r}0.24 \\
{[4]}\end{array}$ & $\begin{array}{r}7.01 \\
{[5]}\end{array}$ & $\begin{array}{r}0.58 \\
{[2]}\end{array}$ & $\begin{array}{r}0.39 \\
\text { [9] }\end{array}$ & $\begin{array}{r}0.27 \\
{[2]}\end{array}$ & $\begin{array}{l}3.68 \\
{[15]}\end{array}$ & $\begin{array}{l}1.6 \\
{[7]}\end{array}$ & 7.6 & High \\
\hline $\begin{array}{l}\text { SBW13 } \\
{[15.83]}\end{array}$ & $\begin{array}{r}4.28 \\
{[1]}\end{array}$ & $\begin{array}{c}10.68 \\
{[1]}\end{array}$ & $\begin{array}{r}3.47 \\
\text { [9] }\end{array}$ & $\begin{array}{l}0.11 \\
{[14]}\end{array}$ & $\begin{array}{r}8.32 \\
{[2]}\end{array}$ & $\begin{array}{l}0.97 \\
{[12]}\end{array}$ & $\begin{array}{l}0.48 \\
{[14]}\end{array}$ & $\begin{array}{l}0.73 \\
{[11]}\end{array}$ & $\begin{array}{r}1.36 \\
{[2]}\end{array}$ & $\begin{array}{r}1.43 \\
{[2]}\end{array}$ & 6.8 & Very High \\
\hline $\begin{array}{l}\text { SBW14 } \\
{[16.97]}\end{array}$ & $\begin{array}{l}4.1 \\
{[2]}\end{array}$ & $\begin{array}{r}9.36 \\
{[2]}\end{array}$ & $\begin{array}{l}3.42 \\
{[10]}\end{array}$ & $\begin{array}{l}0.12 \\
{[13]}\end{array}$ & $\begin{array}{r}6.48 \\
{[8]}\end{array}$ & $\begin{array}{l}0.7 \\
{[10]}\end{array}$ & $\begin{array}{r}0.35 \\
{[7]}\end{array}$ & $\begin{array}{r}0.38 \\
\text { [9] }\end{array}$ & $\begin{array}{l}2.6 \\
\text { [5] }\end{array}$ & $\begin{array}{l}1.69 \\
{[10]}\end{array}$ & 7.6 & High \\
\hline $\begin{array}{l}\text { SBW15 } \\
{[40.12]}\end{array}$ & $\begin{array}{l}2.33 \\
{[12]}\end{array}$ & $\begin{array}{l}3.76 \\
{[13]}\end{array}$ & $\begin{array}{r}3.85 \\
{[7]}\end{array}$ & $\begin{array}{r}0.21 \\
{[6]}\end{array}$ & $\begin{array}{c}4.6 \\
{[12]}\end{array}$ & $\begin{array}{r}0.67 \\
{[8]}\end{array}$ & $\begin{array}{l}0.46 \\
{[12]}\end{array}$ & $\begin{array}{r}0.35 \\
{[7]}\end{array}$ & $\begin{array}{r}2.83 \\
{[8]}\end{array}$ & $\begin{array}{r}1.47 \\
{[4]}\end{array}$ & 8.9 & Medium \\
\hline $\begin{array}{c}\text { SBW16 } \\
{[90.1]}\end{array}$ & $\begin{array}{l}2.9 \\
{[8]}\end{array}$ & $\begin{array}{l}4.58 \\
\text { [9] }\end{array}$ & $\begin{array}{r}4.23 \\
{[5]}\end{array}$ & $\begin{array}{r}0.17 \\
{[8]}\end{array}$ & $\begin{array}{r}6.86 \\
{[6]}\end{array}$ & $\begin{array}{l}0.6 \\
{[4]}\end{array}$ & $\begin{array}{r}0.31 \\
{[5]}\end{array}$ & $\begin{array}{r}0.28 \\
{[3]}\end{array}$ & $\begin{array}{l}3.46 \\
{[13]}\end{array}$ & $\begin{array}{l}1.78 \\
{[12]}\end{array}$ & 7.3 & High \\
\hline $\begin{array}{l}\text { SBW17 } \\
{[94.07]}\end{array}$ & $\begin{array}{l}1.37 \\
{[15]}\end{array}$ & $\begin{array}{l}2.15 \\
{[15]}\end{array}$ & $\begin{array}{l}3.19 \\
{[13]}\end{array}$ & $\begin{array}{r}0.36 \\
{[3]}\end{array}$ & $\begin{array}{l}4.08 \\
{[14]}\end{array}$ & $\begin{array}{r}0.59 \\
{[3]}\end{array}$ & $\begin{array}{l}0.47 \\
{[13]}\end{array}$ & $\begin{array}{r}0.28 \\
{[3]}\end{array}$ & $\begin{array}{l}3.54 \\
{[14]}\end{array}$ & $\begin{array}{r}1.44 \\
{[3]}\end{array}$ & 9.6 & Low \\
\hline
\end{tabular}




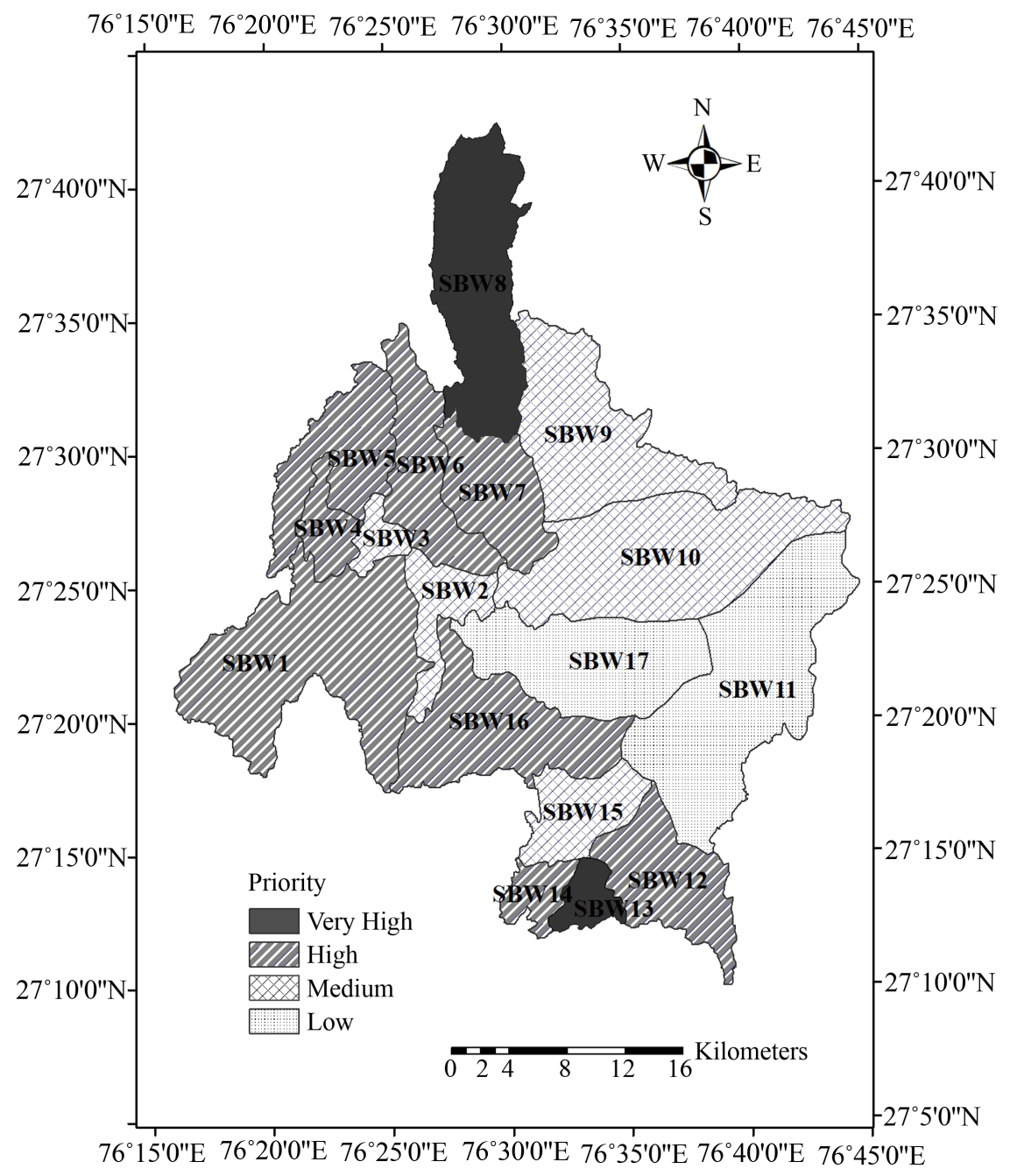

Figure 4. Sub-watershed priority ranking map based on Morphometric analysis.

\subsection{Prioritization of Sub-Watersheds Based on LULC Analysis}

Five LULC categories namely cultivated land, dense forest, open forest, open scrub and wasteland were considered for sub-watershed prioritization in Ruparel watershed. Sub-watersheds with a higher percentage of wasteland or a lower percentage of cultivated land, dense forest, open forest, and open scrub were given high priority, whereas those with a lower percentage of wasteland or a higher percentage of cultivated land, dense forest, open forest, and open scrub were given low priority [8]. Based on the $(\mathrm{Cp})$ value, sub-watersheds were categorized into Very High, High, Medium and Low priority classes. Results show SBW6 and SBW10 fall under Very High priority category, SBW2, SBW5, SBW7, SBW8, SBW9, SBW12, SBW13 and SBW16 fall under High priority, SBW1, SBW3, SBW4 and SBW17 under Medium priority, whereas SBW11, SBW14 and SBW15 fall under low priority category (Table 4 and Figure 5).

The findings of both the morphometric and LULC analysis were then compared to identify the common sub-watersheds that fall within each priority class 
Table 4. Sub-watershed wise priority ranking based on LULC analysis.

\begin{tabular}{|c|c|c|c|c|c|c|c|}
\hline \multirow[b]{2}{*}{ Sub-watersheds } & \multicolumn{7}{|c|}{ Land use and Land cover Analysis } \\
\hline & $\begin{array}{c}\text { Wasteland } \\
{[\%]}\end{array}$ & $\begin{array}{c}\text { Cultivated } \\
\text { land } \\
{[\%]}\end{array}$ & $\begin{array}{c}\text { Dense } \\
\text { forest } \\
{[\%]}\end{array}$ & $\begin{array}{c}\text { Open } \\
\text { forest } \\
{[\%]}\end{array}$ & $\begin{array}{c}\text { Open } \\
\text { Scrub } \\
{[\%]}\end{array}$ & $\begin{array}{c}\mathrm{Cp} \\
\text { value }\end{array}$ & Priority \\
\hline $\begin{array}{c}\text { SBW1 } \\
{[154.76]}\end{array}$ & $\begin{array}{c}17.05 \\
{[6]}\end{array}$ & $\begin{array}{c}12.05 \\
\text { [9] }\end{array}$ & $\begin{array}{r}46.17 \\
{[11]}\end{array}$ & $\begin{array}{r}6.82 \\
\text { [9] }\end{array}$ & $\begin{array}{r}6.48 \\
\text { [9] }\end{array}$ & 8.8 & Medium \\
\hline $\begin{array}{l}\text { SBW2 } \\
{[31.35]}\end{array}$ & $\begin{array}{c}23.36 \\
{[3]}\end{array}$ & $\begin{array}{l}7.31 \\
{[4]}\end{array}$ & $\begin{array}{r}54.31 \\
{[13]}\end{array}$ & - & $\begin{array}{c}8.0 \\
{[11]}\end{array}$ & 7.75 & High \\
\hline $\begin{array}{l}\text { SBW3 } \\
\text { [12.67] }\end{array}$ & $\begin{array}{c}12.86 \\
{[7]}\end{array}$ & $\begin{array}{r}10.9 \\
{[8]}\end{array}$ & $\begin{array}{c}45.15 \\
\text { [9] }\end{array}$ & $\begin{array}{r}5.29 \\
{[6]}\end{array}$ & $\begin{array}{r}20.36 \\
{[14]}\end{array}$ & 8.8 & Medium \\
\hline $\begin{array}{l}\text { SBW4 } \\
{[20.4]}\end{array}$ & - & $\begin{array}{r}3.38 \\
{[1]}\end{array}$ & $\begin{array}{c}31.18 \\
{[6]}\end{array}$ & $\begin{array}{r}33.77 \\
{[11]}\end{array}$ & $\begin{array}{r}29.95 \\
{[17]}\end{array}$ & 8.75 & Medium \\
\hline $\begin{array}{l}\text { SBW5 } \\
{[60.41]}\end{array}$ & $\begin{array}{c}30.79 \\
{[1]}\end{array}$ & $\begin{array}{l}19.2 \\
{[11]}\end{array}$ & $\begin{array}{r}7.45 \\
{[2]}\end{array}$ & $\begin{array}{r}11.41 \\
{[10]}\end{array}$ & $\begin{array}{r}22.15 \\
{[16]}\end{array}$ & 8.0 & High \\
\hline $\begin{array}{c}\text { SBW6 } \\
{[58.17]}\end{array}$ & $\begin{array}{c}19.39 \\
{[5]}\end{array}$ & $\begin{array}{r}6.95 \\
{[2]}\end{array}$ & $\begin{array}{r}64.19 \\
{[14]}\end{array}$ & $\begin{array}{r}0.07 \\
{[1]}\end{array}$ & $\begin{array}{l}4.95 \\
{[7]}\end{array}$ & 5.8 & $\begin{array}{l}\text { Very } \\
\text { High }\end{array}$ \\
\hline $\begin{array}{l}\text { SBW7 } \\
\text { [52.43] }\end{array}$ & $\begin{array}{c}20.45 \\
{[4]}\end{array}$ & $\begin{array}{r}8.37 \\
{[5]}\end{array}$ & $\begin{array}{r}50.16 \\
{[12]}\end{array}$ & $\begin{array}{r}5.07 \\
{[5]}\end{array}$ & $\begin{array}{l}9.65 \\
{[12]}\end{array}$ & 7.6 & High \\
\hline $\begin{array}{c}\text { SBW8 } \\
{[105.81]}\end{array}$ & $\begin{array}{c}24.46 \\
{[2]}\end{array}$ & $\begin{array}{r}18.13 \\
{[10]}\end{array}$ & $\begin{array}{c}34.38 \\
{[7]}\end{array}$ & - & $\begin{array}{l}7.07 \\
{[10]}\end{array}$ & 7.25 & High \\
\hline $\begin{array}{c}\text { SBW9 } \\
{[102.66]}\end{array}$ & $\begin{array}{l}6.41 \\
{[11]}\end{array}$ & $\begin{array}{r}30.11 \\
{[14]}\end{array}$ & $\begin{array}{c}38.48 \\
{[8]}\end{array}$ & $\begin{array}{r}3.52 \\
{[4]}\end{array}$ & $\begin{array}{r}0.89 \\
{[3]}\end{array}$ & 8.0 & High \\
\hline $\begin{array}{l}\text { SBW10 } \\
\text { [143.22] }\end{array}$ & $\begin{array}{l}7.28 \\
{[10]}\end{array}$ & $\begin{array}{r}59.01 \\
{[17]}\end{array}$ & $\begin{array}{r}3.39 \\
{[1]}\end{array}$ & $\begin{array}{r}0.09 \\
{[2]}\end{array}$ & $\begin{array}{r}1.98 \\
{[4]}\end{array}$ & 6.8 & $\begin{array}{l}\text { Very } \\
\text { High }\end{array}$ \\
\hline $\begin{array}{l}\text { SBW11 } \\
{[154.62]}\end{array}$ & $\begin{array}{l}3.07 \\
{[13]}\end{array}$ & $\begin{array}{r}55.66 \\
{[16]}\end{array}$ & - & - & $\begin{array}{r}0.25 \\
{[1]}\end{array}$ & 10.0 & Low \\
\hline $\begin{array}{l}\text { SBW12 } \\
{[62.17]}\end{array}$ & $\begin{array}{l}1.38 \\
{[15]}\end{array}$ & $\begin{array}{r}25.82 \\
{[12]}\end{array}$ & $\begin{array}{c}10.81 \\
{[3]}\end{array}$ & - & $\begin{array}{r}0.76 \\
{[2]}\end{array}$ & 8.0 & High \\
\hline $\begin{array}{l}\text { SBW13 } \\
{[15.83]}\end{array}$ & - & $\begin{array}{r}7.14 \\
{[3]}\end{array}$ & $\begin{array}{r}65.55 \\
{[15]}\end{array}$ & $\begin{array}{l}5.31 \\
{[7]}\end{array}$ & $\begin{array}{l}4.55 \\
{[6]}\end{array}$ & 7.75 & High \\
\hline $\begin{array}{l}\text { SBW14 } \\
\text { [16.97] }\end{array}$ & $\begin{array}{l}1.53 \\
{[14]}\end{array}$ & $\begin{array}{r}9.07 \\
{[6]}\end{array}$ & $\begin{array}{r}46.02 \\
{[10]}\end{array}$ & $\begin{array}{r}6.42 \\
{[8]}\end{array}$ & $\begin{array}{r}20.68 \\
{[15]}\end{array}$ & 10.6 & Low \\
\hline $\begin{array}{l}\text { SBW15 } \\
{[\mathbf{4 0 . 1 2}]}\end{array}$ & $\begin{array}{r}9.07 \\
\text { [9] }\end{array}$ & $\begin{array}{r}27.99 \\
{[13]}\end{array}$ & $\begin{array}{c}29.06 \\
{[5]}\end{array}$ & - & $\begin{array}{r}10.44 \\
{[13]}\end{array}$ & 10.0 & Low \\
\hline $\begin{array}{c}\text { SBW16 } \\
\text { [90.1] }\end{array}$ & $\begin{array}{c}11.06 \\
{[8]}\end{array}$ & $\begin{array}{r}9.74 \\
{[7]}\end{array}$ & $\begin{array}{r}70.81 \\
{[16]}\end{array}$ & $\begin{array}{r}0.09 \\
{[2]}\end{array}$ & $\begin{array}{r}2.11 \\
{[5]}\end{array}$ & 7.6 & High \\
\hline $\begin{array}{l}\text { SBW17 } \\
\text { [94.07] }\end{array}$ & $\begin{array}{l}5.49 \\
{[12]}\end{array}$ & $\begin{array}{r}42.77 \\
{[15]}\end{array}$ & $\begin{array}{c}25.31 \\
{[4]}\end{array}$ & $\begin{array}{r}0.21 \\
{[3]}\end{array}$ & $\begin{array}{r}5.51 \\
{[8]}\end{array}$ & 8.4 & Medium \\
\hline
\end{tabular}

(Table 5). It was concluded that SBW5, SBW7, SBW12 and SBW16 are the common sub-watersheds that fall under High priority, SBW3 falls under Medium priority whereas SBW11 falls under Low priority, based on morphometric and LULC analysis. The remaining eleven sub-watersheds show very less or no co-relation under morphometric and LULC analysis. Figure 6 presents the 


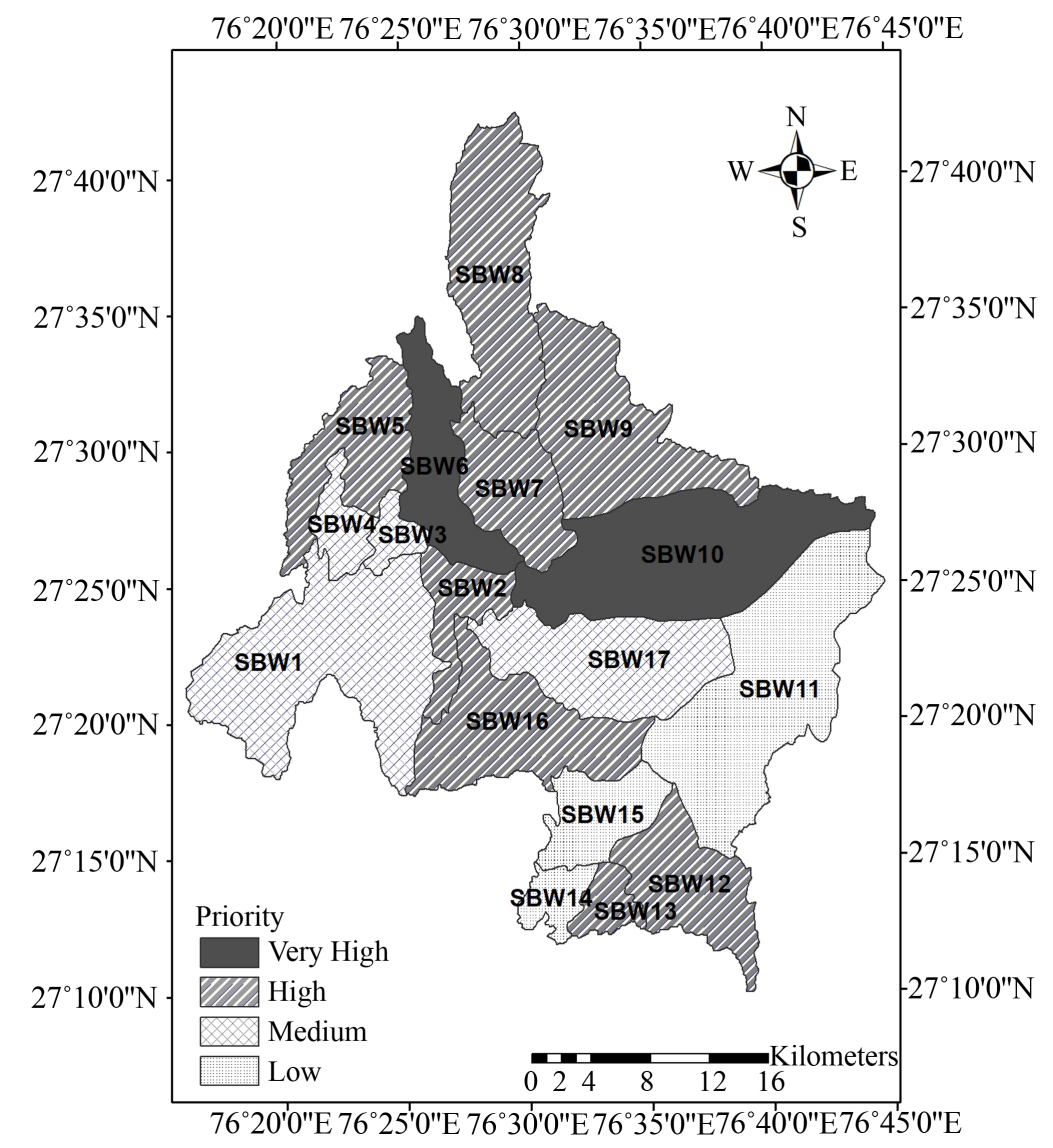

Figure 5. Sub-watershed priority ranking map based on LULC analysis.

Table 5. Sub-watershed wise common priority ranking in Ruparel watershed.

\begin{tabular}{|c|c|c|c|c|c|}
\hline Sub-watersheds & $\begin{array}{c}\text { (Cp) value for Morphometric } \\
\text { Parameters }\end{array}$ & Final Priority & $\begin{array}{c}\text { (Cp) value for } \\
\text { LU/LC parameters }\end{array}$ & $\begin{array}{l}\text { Final } \\
\text { Priority }\end{array}$ & $\begin{array}{l}\text { Common } \\
\text { Priority }\end{array}$ \\
\hline SBW1 & 7.4 & High & 8.8 & Medium & \\
\hline SBW2 & 9.1 & Medium & 7.75 & High & \\
\hline SBW3 & 8.8 & Medium & 8.8 & Medium & Medium \\
\hline SBW4 & 8.2 & High & 8.75 & Medium & \\
\hline SBW5 & 7.5 & High & 8.0 & High & High \\
\hline SBW6 & 7.6 & High & 5.8 & Very High & \\
\hline SBW7 & 8.0 & High & 7.6 & High & High \\
\hline SBW8 & 6.1 & Very High & 7.25 & High & \\
\hline SBW9 & 8.5 & Medium & 8.0 & High & \\
\hline SBW10 & 9.0 & Medium & 6.8 & Very High & \\
\hline SBW11 & 10.5 & Low & 10.0 & Low & Low \\
\hline SBW12 & 7.6 & High & 8.0 & High & High \\
\hline SBW13 & 6.8 & Very High & 7.75 & High & \\
\hline SBW14 & 7.6 & High & 10.6 & Low & \\
\hline SBW15 & 8.9 & Medium & 10.0 & Low & \\
\hline SBW16 & 7.3 & High & 7.6 & High & High \\
\hline SBW17 & 9.6 & Low & 8.4 & Medium & \\
\hline
\end{tabular}




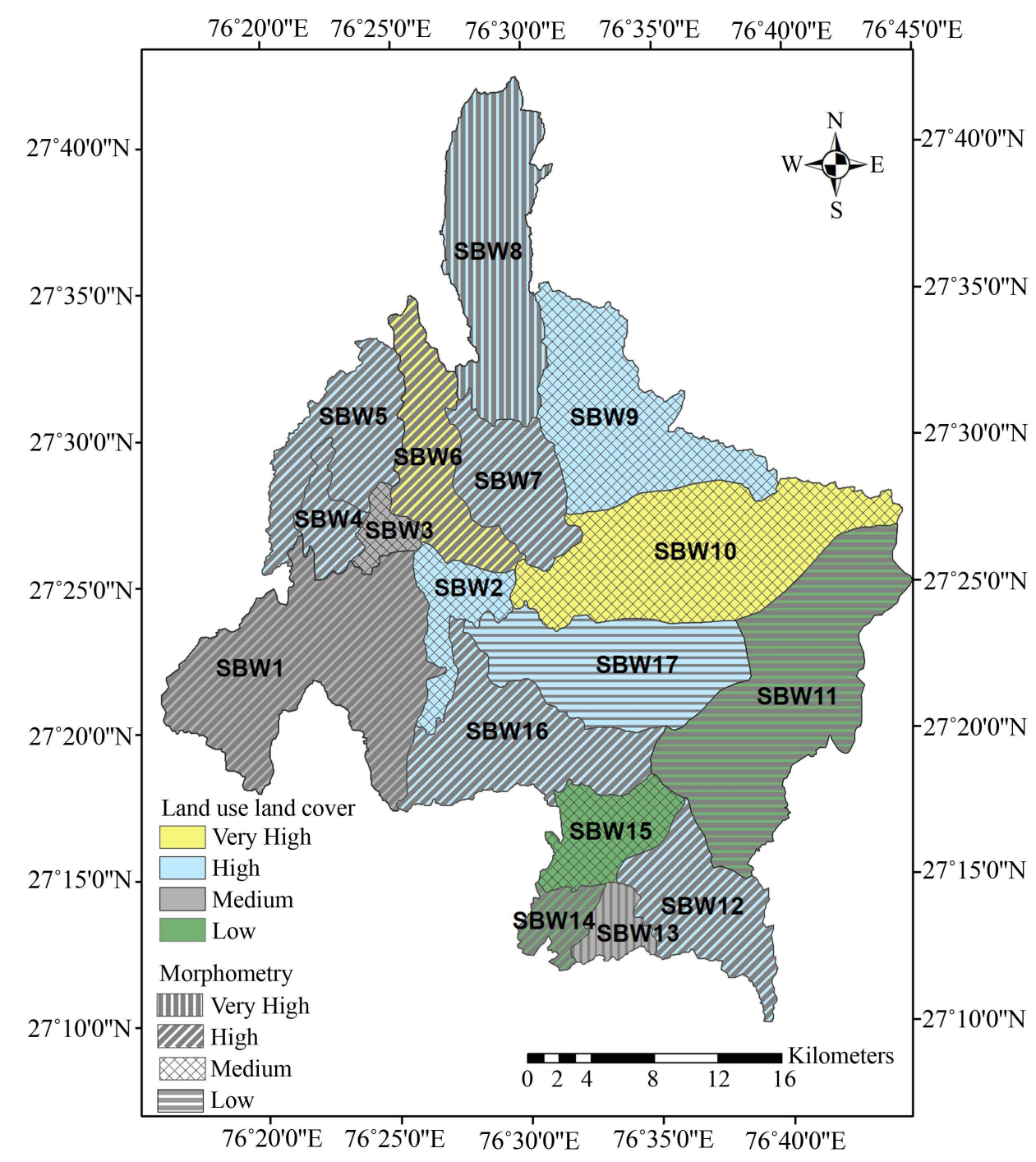

Figure 6. Sub-watershed wise final prioritiy ranking map based on superimposition of Morphometric and LULC parameters.

composite sub-watershed prioritization, on the basis of integration of both morphometric and LULC parameters after their superimpostion in GIS.

\section{Conclusion}

The current study illustrates the holistic approach of remote sensing and GIS techniques for the prioritization of sub-watersheds in Ruparel watershed based on drainage morphometry and LULC analysis. Basin morphometry in integration with LULC analysis enables prioritization and characterization at sub-watershed level giving a measure of risk potential of sub-watersheds. The present study indicates the stress of the sub-watersheds within the rainfed Ruparel watershed. Correlation of results based on morphometric and LULC analysis show SBW5, SBW7, SBW12 and SBW16 fall under High priority and demand prompt attention from planners and decision makers for conservation measures. The sub-watersheds, SBW3 falls under Medium priority, whereas SBW11 falls under 
Low priority category. The remaining sub-watersheds show little or no correlation and therefore differ in their priority. Sub-watersheds with High priority ranking need to be taken up for immediate conservation measures for sustainable development under watershed development programme of the state/central governments.

\section{Acknowledgements}

Authors express their gratitude to the Chairman, Department of Geology, Aligarh Muslim University, Aligarh for providing required infrastructural facilities at the Remote sensing lab. Thanks are due to National Remote Sensing Centre (NRSC), Hyderabad for providing satellite data and USGS website for freely available ASTER data.

\section{Conflicts of Interest}

The authors declare no conflicts of interest regarding the publication of this paper.

\section{References}

[1] Khanday, M.Y. and Javed, A. (2016) Prioritization of Sub-Watersheds for Conservation Measures in a Semi-Arid Watershed Using Remote Sensing and GIS. Journal of the Geological Society of India, 2, 185-196.

https://doi.org/10.1007/s12594-016-0477-7

[2] Chakraborty, A.K. (1991) Sediment Yield Prediction and Prioritization of Watersheds Using Remote Sensing. http://www.gisdevelopment.net/aars/acrs/1991/psq/ps003.shtml

[3] Biswas, S., Sudhakar, S. and Desai, V.R. (1999) Prioritization of Sub-Watersheds Based on Morphometric Analysis of Drainage Basin, District Midnapore, West Bengal. Journal of the Indian Society of Remote Sensing, 27, 155-166.

https://doi.org/10.1007/BF02991569

[4] Nooka Ratnam, K., Srivastava, Y.K., Venkateshwara Rao, V., Amminedu, E. and Murthy, K.S.R. (2005) Check Dam Positioning by Prioritization of Micro-Watersheds Using SYI Model and Morphometric Analysis-Remote Sensing and GIS Perspective. Journal of the Indian Society of Remote Sensing, 33, 25-38. https://doi.org/10.1007/BF02989988

[5] Javed, A., Khanday, M.Y. and Ahmed, R. (2009) Prioritization of Sub-Watersheds Based on Morphometric and Land Use Analysis Using Remote Sensing and GIS Techniques. Journal of the Indian Society of Remote Sensing, 37, Article No. 261. https://doi.org/10.1007/s12524-009-0016-8

[6] Patel, D.P., Dholakia, M.B., Naresh, N. and Srivastava, P.K. (2012) Water Harvesting Structure Positioning by Using Geo-Visualization Concept and Prioritization of Mini-Watersheds through Morphometric Analysis in the Lower Tapi Basin. Journal of the Indian Society of Remote Sensing, 40, 299-312.

https://doi.org/10.1007/s12524-011-0147-6

[7] Malik, A., Kumar, A. and Kandpal, H. (2019). Morphometric Analysis and Prioritization of Sub-Watersheds in a Hilly Watershed Using Weighted Sum Approach. Arabian Journal of Geosciences, 4, 118. https://doi.org/10.1007/s12517-019-4310-7

[8] Javed, A., Khanday, M.Y. and Rais, S. (2011) Watershed Prioritization Using Mor- 
phometric and Land Use/Land Cover Parameters: A Remote Sensing and GIS Based Approach. Journal of the Geological Society of India, 78, 63-75. https://doi.org/10.1007/s12594-011-0068-6

[9] Magesh, N.S., Jitheshlal, K.V., Chandrasekar, N. and Jini, K.V. (2013) Geographical Information System Based Morphometric Analysis of Bharathapuzha River Basin, Kerala, India. Applied Water Science, 3, 467-477. https://doi.org/10.1007/s13201-013-0095-0

[10] Nautiyal, M.D. (1994) Morphometric Analysis of a Drainage Basin Using Aerial Photographs: A Case Study of Khairkuli Basin, District Dehradun, UP. Journal of the Indian Society of Remote Sensing, 22, 252-262. https://doi.org/10.1007/BF03026526

[11] Strahler, A.N. (1964) Quantitative Geomorphology of Drainage Basins and Channel Networks. In: Chow, V.T., Ed., Handbook of Applied Hydrology, McGraw Hill Book Company, New York, Section 4-11.

[12] Reddy, O.G.P., Maji, A.K. and Gajbhiye, S.K. (2004) Drainage Morphometry and Its Influence on Landform Characteristics in a Basaltic Terrain, Central India-A Remote Sensing and GIS Approach. The International Journal of Applied Earth $\mathrm{Ob}$ servation and Geoinformation, 6, 1-16. https://doi.org/10.1016/j.jag.2004.06.003

[13] Iqbal, M. and Sajjad, H. (2014) Watershed Prioritization Using Morphometric and Land Use/Land Cover Parameters of Dhudganga Catchment Kashmir Valley India Using Spatial Technology. Journal of Geophysics and Remote Sensing, 3, 12-23.

[14] Horton, R.E. (1932) Drainage-Basin Characteristics. Eos, Transactions American Geophysical Union, 13, 350-361. https://doi.org/10.1029/TR013i001p00350

[15] Schumm, S.A. (1956) Evolution of Drainage Systems and Slope in Badlands at Perth Amboy, New Jersey. GSA Bulletin, 67, 597-646. https://doi.org/10.1130/0016-7606(1956)67[597:EODSAS]2.0.CO;2

[16] Horton, R.E. (1945) Erosional Development of Streams and Their Drainage Basins: Hydro Physical Approach to Quantitative Morphology. Geological Society of American Bulletin, 55, 275-370. https://doi.org/10.1130/0016-7606(1945)56[275:EDOSAT]2.0.CO;2

[17] Smith, K.G. (1950) Standards for Grading Texture of Erosional Topography. American Journal of Science, 248, 655-688. https://doi.org/10.2475/ajs.248.9.655

[18] Strahler, A.N. (1957) Quantitative Analysis of Watershed Geomorphology. Eos, Transactions American Geophysical Union, 38, 913-920. https://doi.org/10.1029/TR038i006p00913

[19] Sreedhar, Y., Nagaraju, A. and Krishna, G.M. (2016) An Appraisal of Land Use/Land Cover Change Scenario of Tummalapalle, Cuddapah Region, India-A Remote Sensing and GIS Perspective. Advances in Remote Sensing, 5, 232-245. https://doi.org/10.4236/ars.2016.54019

[20] Wasteland Atlas of India (2019) Department of Land Resources. Ministry of Rural Development Government of India, New Delhi and National Remote Sensing Centre, Indian Space Research Organisation. Department of Space, Government of India, Hyderabad. 